\title{
FIXED POINTS FOR CERTAIN NONCONTINUOUS TRANSFORMATIONS ${ }^{1}$
}

\author{
O. H. HAMILTON
}

Professor John Nash [1] has defined a connectivity map from a space $A$ into a space $B$ as a mapping $T$ such that the induced map $g$ of $A$ into $A \times B$ defined by $g(p)=[p \times T(p)]$ transforms connected subsets of $A$ onto connected subsets of $A \times B$. He has inquired whether or not every connectivity map of a closed $n$-cell into itself has a fixed point. It is the purpose of this paper to answer his question in the affirmative and to develop some other properties of connectivity maps.

We shall make use of the properties of a closed $n$-cell given by the following:

Proposition A. If p and $q$ are two points of a closed $n$-cell I separated by a subset $N$ of $I$, then some connected subset $N_{1}$ of $N$ separates $p$ from $q$ in $I$.

Proposition B. If $K$ is any subcontinuum of a closed $n$-cell I then the boundary of every complementary domain of $K$ is connected.

It follows by a theorem of Borsuk [2, p. 188, Corollary 32] that the closed $n$-cell $I$ is unicoherent; that is, if $I$ is expressed as the union of two of its closed and connected subsets $H$ and $K$, then $H \cap K$ is connected. Furthermore, it is well known [4, p. 51, Theorem 4.12] that in a connected and locally connected space, and hence in $I$, unicoherence is equivalent to each of the properties given by Propositions $\mathrm{A}$ and $\mathrm{B}$.

We prove the following:

THEOREM 1. If $T$ is a connectivity map of a Hausdorff space A onto a Hausdorff space $B, p$ is a point of $A, V$ and $U$ are open sets containing $p$ and $T(p)$ respectively, then every nondegenerate connected subset of $A$ containing $p$ contains a point $q$ of $V$ distinct from $p$ such that $T(q) \in U$.

Proof. Suppose $E$ is a nondegenerate connected subset of $A$ containing $p$ but no point $q$ of $V$ distinct from $p$ such that $T(q) \in U$. Then $g(E)$ is the union of two mutually separated sets $g(p)$

Presented to the Society, November 23, 1956; received by the editors August 4, 1956 and, in revised form, November 12, 1956.

1 The research upon which this paper is based was done in part with the aid of a grant from The Research Corporation. 
$=[p \times T(p)]$ and $g[E-\{p\}]$ since $U \times V$ contains $[p \times T(p)]$ but no point of $g[E-\{p\}]$.

Theorem 2. If $T$ is a connectivity map of a Hausdorff space $A$ into a Hausdorff space $B$ and $C$ is a closed subset of $B$ then each component of $T^{-1}(C)$ is closed.

Proof. Suppose some component $E$ of $T^{-1}(C)$ is not closed. Then $E$ has a limit point $p$ which does not belong to it and hence $T(p)$ does not belong to $C$. Then since $C$ is closed there is an open subset $U$ of $B$ containing $T(p)$ but no point of $C$. Hence $T^{-1}(U) \cap E=0$. This contradicts the fact that, by Theorem 1, the connected set $E \cup\{p\}$ must contain a point $q$ distinct from $p$ such that $T(q) \in U$. Hence $E$ is closed.

Corollary. If $T$ is a connectivity map of a Hausdorff space $A$ into a Hausdorff space $B, p$ is a point of $A, U$ is an open subset of $B$ containing $T(p), C$ is the subset of $A$ consisting of all points $q$ of $A$ such that $T(q) \in \bar{U}$, then each component $E$ of $C$ is closed.

Definition. If $T$ is a mapping of a subset $A$ of a space $S$ onto a subset $B$ of $S, T$ will be said to be peripherally continuous if and only if for each point $p$ of $A$ and each pair of open sets $U$ and $V$ containing $T(p)$ and $p$ respectively there is an open set $D \subset V$ containing $p$ such that $T$ transforms the boundary $F$ of $D$ into $U$.

TheOREM 3. If $T$ is a connectivity map of a closed $n$-cell $I, n \geqq 2$, onto a subset $B$ of $I$, then $T$ is peripherally continuous on $I$. Furthermore if $p$ is any point of $I$ and $U$ and $V$ are open subsets of $I$ containing $T(p)$ and $p$ respectively there is a connected open set $D$ of $I$ with connected boundary $F$ such that $p \in D, D \cup F \subset V$, and $T(F) \subset U$.

Proof. We may take $I$ to be a closed spherical $n$-cell with radius $r$. Let $p$ be any point of $I$. Let $U$ and $V$ be open subsets of $I$ containing $T(p)$ and $p$ respectively and let $U_{1}$ and $V_{1}$ be open subsets of $U$ and $V$ respectively containing $T(p)$ and $p$ respectively such that $\bar{V}_{1} \subset V$ and $\bar{U}_{1} \subset U$. Without loss of generality we may take $\bar{V}, \bar{V}_{1}, \bar{U}$, and $\bar{U}_{1}$ to be closed topological $n$-cells each the intersection of $I$ and a closed spherical $n$-cell in $n$-space with $p$ as center, and hence each having, with respect to $I$, a connected boundary. Let $x$ be a point of the boundary of $V$ with respect to $I$.

Let $N$ be the subset of $\bar{V}_{1}$ consisting of all points $q$ such that $T(q) \in \bar{U}_{1}$ and let $\left\{G_{\alpha}\right\}$ be the collection of all components of $N$. Since $\bar{V}_{1}$ is closed and since by the corollary to Theorem 2 each component of $T^{-1}\left(\bar{U}_{1}\right)$ is closed it follows that each element $g$ of $\left\{G_{\alpha}\right\}$ is closed. 
For each element $g$ of the collection $\left\{G_{\alpha}\right\}$ let $g^{\prime}$ be the union of $g$ and all its complementary domains in $V$ which are subsets of $\bar{V}_{1},\left\{G_{\alpha}^{\prime}\right\}$ the collection of all these sets and $L$ the union of the sets of $\left\{G_{\alpha}^{\prime}\right\}$. Since $\bar{V}_{1}$ is closed, it follows that each $g^{\prime}$ is closed. Note (1) that no $g^{\prime}$ separates $\bar{V}$ since by definition of $g^{\prime}$, its only complementary domain with respect to $\bar{V}$ is the connected subset of $\bar{V}-g^{\prime}$ which contains the connected set $\bar{V}-\bar{V}_{1}$.

Then $p$ is an interior point of some element $g^{\prime}$ of $\left\{G_{\alpha}^{\prime}\right\}$. For suppose $p$ is on the boundary with respect to $I$ of each element $g^{\prime}$ which contains it. Since the boundary with respect to $I$ of $g^{\prime}$ is an element $g$ of $\left\{G_{\alpha}\right\}$ and a component of $\bar{V}_{1} \cap T^{-1}\left(\bar{U}_{1}\right)$, then (2) $p$ can be contained in only one such element and (3) $p$ must be a limit point of $\bar{V}-L$. The set $L$ separates some two points $c$ and $d$ of $\bar{V}$ for if not, $(\bar{V}-L)$ $\cup\{p\}$ is a connected subset of $\bar{V}$ containing the point $x$ of $\bar{V}-V$ and $p$ but no point $q$ of $\bar{V}_{1}$ distinct from $p$ such that $T(q) \in \bar{U}_{1}$, and this contradicts Theorem 1 . Hence, by Proposition A, some connected subset $M$ of $L$ separates $c$ from $d$ in the closed $n$-cell $\bar{V}$. Then $M$ is a subset of some element $g^{\prime}$ of $\left\{G_{\alpha}^{\prime}\right\}$ which separates $c$ from $d$ in $\bar{V}$, and this contradicts (1) above. Hence the assumption that $p$ is not an interior point of some element $g_{p}^{\prime}$ of $\left\{G_{\alpha}^{\prime}\right\}$ is false. Let $g$ be the element of $\left\{G_{\alpha}\right\}$ which is the boundary of the element $g_{p}^{\prime}$ of $\left\{G_{\alpha}^{\prime}\right\}$ which contains $p$ as an interior point and let $D$ be the maximum connected domain of $\bar{V}_{1}-g$ which contains $p$. Since $g$ is a compact continuum it follows from Proposition B that the boundary $F$ of $D$ is a connected subset of $g$. Furthermore by definition of $g$ and $D, F \cup D$ $\subset \bar{V}_{1} \subset V$. Hence $D$ is the connected open set required by the statement of the theorem and furthermore $T$ is then, by definition, peripherally continuous.

It is not known whether or not a peripherally continuous transformation of a closed $n$-cell, $n \geqq 2$, into itself is necessarily a connectivity map. The following is an example of a peripherally continuous transformation of the closed 1 -cell, $0 \leqq x \leqq 1$, into itself which is not a connectivity map and which furthermore leaves no point fixed.

EXAmple 1. On $0 \leqq x \leqq 1$ for $x$ rational let $T(x)=\pi / 4$ and for $x$ irrational let $T(x)=3 / 4$.

THEOREM 4. Let $T$ be a peripherally continuous transformation of a closed $n$-cell $I, n \geqq 2$ into itself. Let it be assumed that $I$ is the closed $n$ cube consisting of the points $\left(x_{1}, x_{2}, \cdots, x_{n}\right)$ given by the inequalities $0 \leqq x_{i} \leqq 1$. Let the faces $x_{i}=0$ and $x_{i}=1$ be designated by $A_{i}$ and $B_{i}$ respectively. If $x$ is the point $\left(x_{1}, x_{2}, \cdots, x_{n}\right)$ let $T(x)=x^{\prime}$ be the point $\left(x_{1}^{\prime}, x_{2}^{\prime}, \cdots, x_{n}^{\prime}\right)$. For each $i, 1 \leqq i \leqq n$, let $M_{i}, L_{i}$, and $N_{i}$ designate the 
subsets of I for which $x_{i}^{\prime} \leqq x_{i}, x_{i}^{\prime}=x_{i}$, and $x_{i}^{\prime} \geqq x_{i}$ respectively. Then the components of $M_{i}, L_{i}$, and $N_{i}$ are closed and if $q\left(q_{1}, q_{2}, \cdots, q_{n}\right)$ is a point in the common boundary between a component $E$ of $M_{i}$ or $N_{i}$ and a connected subset of $I-E$, then $q \in L_{i}$.

Proof. Let $q\left(q_{1}, q_{2}, \cdots, q_{n}\right)$ be a limit point of a component $E$ of $M_{i}$ and suppose that $q$ does not belong to $M_{i}$. Then by definition of $M_{i}, q_{i}^{\prime}=q_{i}+d$ for some $d>0$. Then since $T$ is peripherally continuous there is a connected open set $D$ of diameter $<d / 3$ containing $q$ such that (1) $E-(\bar{D} \cap E) \neq 0$, and (2) if $x$ is a point of $F$, the boundary of $D$, then $\rho[T(x), T(q)]<d / 3$. Then the connected set $E$, since it contains points outside $\bar{D}$ and within $D$, must contain a point $x$ of $F$. That is, $\rho[T(x), T(q)]<d / 3, \rho(x, q)<d / 3$. Hence $\left|x_{i}^{\prime}-q_{i}^{\prime}\right|<d / 3$ and $\left|x_{i}-q_{i}\right|<d / 3$. With $q_{i}^{\prime}=q_{i}+d$, these inequalities give $x_{i}^{\prime}>x_{i}+d / 3$ and this contradicts the fact that $x$ is in $M_{i}$. Hence the assumption that $q$ does not belong to $M_{i}$ is false. Hence $E$ is closed.

It can be shown in the same way that each component of $L_{i}$ or of $N_{i}$ is closed.

Let $q\left(q_{1}, q_{2}, \cdots, q_{n}\right)$ be a point in the common boundary between a component $E$ of $M_{i}$ and some connected subset $R$ of $I-E$, and suppose $q$ does not belong to $L_{i}$. Since $q \in M_{i}, q_{i}=q_{i}^{\prime}+d$ for some $d>0$. Let $\delta$ be a positive real number $<d / 3$ such that a spherical region with center $q$ and diameter $<\delta$ does not contain all of $E$. Then since $T$ is peripherally continuous, it follows by an argument used in the proof of the second part of Theorem 3 that there is a connected domain $D$ with respect to $I$ of diameter $<\delta$ containing $q$ with connected boundary $F$ such that (1) $D$ contains a point $z$ of $R$, (2) $R-(\bar{D} \cap R)$ $\neq 0,(3)$ if $x\left(x_{1}, x_{2}, \cdots, x_{n}\right)$ is in $F$ then $\rho[T(x), T(q)]<d / 3$. Then $\left|x_{i}^{\prime}-q_{i}^{\prime}\right|<d / 3,\left|x_{i}-q_{i}\right|<d / 3$, and since $q_{i}=q_{i}^{\prime}+d$, it follows that $x_{i}>x_{i}^{\prime}$. Hence $x \in M_{i}$ and therefore $F \subset E$. But the connected set $R$ contains a point of $F$ and hence a point of $E$. This contradicts $R \subset I-E$. Hence the assumption that $q$ does not belong to $L_{i}$ is false. Similarly it can be shown that each point common to the boundaries of a component $E$ of $N_{i}$ and a connected subset of $I-E$ is in $L_{i}$.

THEOREM 5. If $T$ is a peripherally continuous transformation of a closed $n$-cell, $n \geqq 2$, into itself, then $T$ leaves a point of I fixed.

Proof. Let $E_{i}$ be the component of $N_{i}$ which contains $A_{i}$. Then by Theorem $4, E_{i}$ is closed. Let $\left\{G_{\alpha}^{1}\right\}$ be the collection of all components of $I-E_{i}$ which contain points of $B_{i}$. Let $H_{i}$ be $\left[\cup_{\alpha} G_{\alpha}^{i}\right] \cup B_{i}$. Then $H_{i}$ is connected since $B_{i}$ is connected and since each $G_{\alpha}^{b}$ is connected and contains a point of $B_{i}$. Let $K_{i}$ be the subset of $E_{i}$ consisting of points in the common boundary between $H_{i}$ and $E_{i}$. Then by 
Theorem $4, K_{i}$ is a closed subset of $L_{i}$ and hence $F_{i}=K_{i} \cup\left(B_{i} \cap L_{i}\right)$ is a closed subset of $L_{i}$. Note: (1) that no component $C$ of $I-F_{i}$ contains points of both $A_{i}$ and $B_{i}$. For suppose $C$ contains a point $a$ of $A_{i}$ and a point $b$ of $B_{i}$. Then $a \in E_{i}$ and $b \in H_{i}$. Hence $C$ contains a point of $K_{i}$, the common boundary between $H_{i}$ and $E_{i}$. This contradicts $K_{i} \subset F_{i}$ and $C \subset I-F_{i}$.

Then let $W$ be a transformation of $I$ into itself defined as follows: For each point $x\left(x_{1}, x_{2}, \cdots, x_{n}\right)$ of $I$ let $W(x)$ be designated by $x^{\prime \prime}\left(x_{1}^{\prime \prime}, x_{2}^{\prime \prime}, \cdots, x_{n}^{\prime \prime}\right)$. (Compare [3, p. 40].) Let $d_{i}(x)=\rho\left(x, F_{i}\right)$. Then (a) if $x$ belongs to a component of $I-F_{i}$ which contains a point of $B_{i}$ and hence by (1) above no point of $A_{i}$, let $x_{i}^{\prime \prime}=x_{i}-1 / 2\left[d_{i}(x) \cdot x_{i}\right]$. Then since $x_{i} \neq 0, x_{i}^{\prime \prime} \neq x_{i}$, (b) if $x \in F_{i}$ let $x_{i}^{\prime \prime}=x_{i}$, and (c) if $x$ belongs to a component of $I-F_{i}$ which contains no point of $B_{i}$, let $x_{i}^{\prime \prime}$ $=x_{i}+1 / 2\left[d_{i}(x) \cdot\left(1-x_{i}\right)\right]$. Since $1-x_{i} \neq 0$, we have in this case as in (a) that $x_{i}^{\prime \prime} \neq x_{i}$.

Then since $d_{i} / 2<1$ we have (2) $0 \leqq x_{i}^{\prime \prime} \leqq 1$, and (3) $x_{i}=x_{i}^{\prime \prime}$ if and only if $x \in F_{i} \subset L_{i}$, that is only if $x_{i}=x_{i}^{\prime}$. The transformation $W$ is, by its definition a continuous mapping of $I$ into itself and hence, by the Brouwer fixed point theorem for $n$-cells, leaves some point $z$ of $I$ fixed. That is for each $i=1,2, \cdots, n, z_{i}^{\prime \prime}=z_{i}$. The point $z$ then is in $\bigcap_{i=1}^{n} L_{i}$ and hence by definition of $L_{i}, z_{i}^{\prime}=z_{i}, i=1,2, \cdots, n$. That is, $T(z)=z$, and $T$, as required, leaves a point of $I$ fixed.

THEOREM 6. If $T$ is a connectivity map of a closed $n$-cell I into itself, $T$ leaves a point of I fixed.

Proof. If $n=1$ and $T$ is a connectivity map of $I, 0 \leqq x \leqq 1$, into itself, $g(I)$, the graph of the mapping is connected by definition of a connectivity map. Furthermore $g(I)$ contains the points $0 \times T(0)$ and $1 \times T(1)$ in the subsets $0 \times I$ and $1 \times I$ respectively. Hence the connected set $g(I)$ contains a point of the connected set $x \times x$ in $I \times I$. That is $T(x)=x$ for some $x \in I$.

If $n \geqq 2$ the Theorem follows directly from Theorems 3 and 5 .

The following is an example of a connectivity map (and hence a peripherally continuous transformation) of a closed $n$-cell, for any $n \geqq 2$, into itself which is not continuous.

EXAMPLE 2. Let $I$ be the closed spherical $n$-cell of radius 1 and center at the origin. Let $(r, \theta)$ designate a point of $I$ with the understanding that if $0<r<1,(r, \theta)$ lies on a straight line segment joining the origin, $(0,0)$, to $(1, \theta)$. Let $C$ be a topological ray with endpoint at $(0,0)$, which has each point of $F$, the boundary of $I$, as a limit point but whose intersection with an $(n-1)$-sphere with radius $r, 0 \leqq r<1$ consists of one and only one point. 
Let $T$ be a transformation of $I$ into itself defined as follows: For each point $x,(r, \theta)$ of $I, 0<r \leqq 1$ let $T(x)$ be the point of $C$ whose distance from the origin is $1-r$. If $(0,0)$ designates the origin $O$, let $T(0,0)$ be a particular point $O^{\prime}=(I, \theta)$ of $F$. Then $T$ and $g$, the induced graph mapping, are continuous except at the origin. Hence any connected subset $N$ of $I-O$ is transformed by $g$ onto a connected subset of $I \times I$. Suppose $N$ is a connected subset of $I$ which contains the origin and that $g(N)$ is the union of two mutually separated sets $A^{\prime}$ and $B^{\prime}$. Let $A$ and $B$ designate $g^{-1}\left(A^{\prime}\right) \cap N$ and $g^{-1}\left(B^{\prime}\right) \cap N$ respectively. One of the sets $A$ and $B$, say $B$, contains a limit point of the other and this point must be $O$. Since $A^{\prime}$ and $B^{\prime}$ are separated there are open sets $V$ and $U$ containing $O$ and $O^{\prime}$ respectively such that $V \times U$ contains $\left(O \times O^{\prime}\right)$, a point of $B^{\prime}$, but no point of $A^{\prime}$, and such that $T^{-1}(U) \subset V$. Then the open set $U$ contains a point $\left(1-r_{1}, \phi\right)$ of $C-[C \cap T(A)]$ such that $A$ contains points both within and exterior to the $(n-1)$-sphere $S$ of radius $r_{1}$. Then $S=T^{-1}\left(1-r_{1}, \phi\right)$. Since $\left(1-r_{1}, \phi\right)$ is not in $T(A), S$ contains no point of $A$. However the subsets $A_{1}$ and $A_{2}$ of $A$ within and exterior to $S$ are each nonvacuous. Then $N$ is the union of the two separated sets $B \cup A_{1}$ and $A_{2}$, and this contradicts the assumption that $N$ is connected. Hence $g(N)$ is connected.

Note that the point $(1 / 2, \phi)$ of $C$ is transformed into itself by $T$.

Suppose $I$ is a closed $n$-cell and $T$ a transformation defined on $I$ having the property that the graph mapping $g(x)=[x \times T(x)]$ transforms each connected and locally connected subset of $I$ onto a connected subset of $I \times I$. This is weaker than requiring that $T$ be a connectivity transformation. Then the example described below shows that $T$ need not leave a point of $I$ invariant.

EXAMPLE 3. Let $I$ be taken as the closed spherical $n$-cell with unit radius and center at the origin. Let the points of the boundary $F$ of $I$ be designated by $(1, \theta)$ and the points of $I$ in general by $(r, \theta)$ with the understanding that $(r, \theta)$ lies on the straight line segment joining $(0,0)$ to $(1, \theta)$ such that $\rho[(r, \theta),(0,0)]=r$. Let $\left\{1, \theta_{i}\right\}, 2 \leqq i<\infty$ be an everywhere dense countable subset of $F$. Let $C$ be a ray which is a subset of the open spherical region $r<1$ with endpoint at $\left(1 / 2, \theta_{2}\right)$ which contains $\left(1-1 / i, \theta_{i}\right), i \geqq 2$, and whose intersection with an $(n-1)$-sphere with center at $(0,0)$ and radius $r, 1 / 2 \leqq r<1$ consists of one and only one point. For each point $(r, \theta), 0 \leqq r<1$ let $T(r, \theta)$ be a point $((r+1) / 2, \phi)$, which is the intersection of $C$ and the $(n-1)$-sphere whose center is $O$ and whose radius is $(r+1) / 2$. For each point $(1, \theta)$ on the boundary of $I$ let $T(1, \theta)$ be the point antipodal to $(1, \theta)$. 
Then it follows from the definition of $T$ that it leaves no point of $I$ fixed. However, we show below that the graph mapping $g$ transforms locally connected and connected subsets of $I$ onto connected subsets of $I \times I$.

For suppose $N$ is a connected and locally connected subset of $I$ such that $g(N)$ is not connected. Let $F$ be the boundary of $I$. Then $N$ contains points of both $F$ and $I-F$ since $T$ and hence $g$ is continuous on $I-F$ and on $F$. Since $g(N)$ is not connected it is the union of two mutually separated sets $A^{\prime}$ and $B^{\prime}$. Let $A$ and $B$ designate $N \cap g^{-1}\left(A^{\prime}\right)$ and $N \cap g^{-1}\left(B^{\prime}\right)$ respectively. Since these sets are disjoint, one of them, say $A$, contains a limit point $p$ of the other, $B$. Then $p$ must be a point of $A \cap F$ which is a limit point of $B-(F \cap B)$, since $T$ and $g$ are each continuous on $F$ and on $I-F$. Let $\epsilon>0$ and $R$ a connected open set of diameter $<\epsilon$ containing $p$. Since $N$ is locally connected there is an open connected subset $V \subset R$ of $N$ containing $p$ and a point $q$ of $B \cap(I-F)$. Let $E$ be the component of $B \cap V$ which contains $q$. Since $A \cap(I-F)$ and $B \cap(I-F)$ are mutually separated it follows that $E$ has a point or limit point in $F \cap V$, and hence that every $(n-1)$-sphere of sufficiently large radius intersects $E$. There is a point $\left(1, \theta_{j}\right)$ of the sequence $\left\{1, \theta_{i}\right\}$ such that the distance from the point $\left(1-1 / j, \theta_{j}\right)$ of $C$ to $T(p)$ in $A^{\prime}$ is $<\epsilon$, and such that the $(n-1)$ sphere $S$ with center at $O$ and radius $1-2 / j$ intersects $E$ in a point $q_{j}$ such that $\rho\left(q_{j}, p\right)<\epsilon$. Then $T\left(q_{j}\right)=\left(1-1 / j, \theta_{j}\right)$. Since $\epsilon$ is arbitrary, we have shown that each open set in $I \times I$ containing the point $[p \times T(p)]$ contains a point of $B^{\prime}$ and hence that $[p \times T(p)] \in A^{\prime}$ is a limit point of $B^{\prime}$. Hence $A^{\prime}$ and $B^{\prime}$ are not mutually separated as assumed and $g(N)$ is connected.

It is easy, on the other hand, to find connected subsets of $I$ which are not transformed into connected subsets of $I \times I$ by $g$. The example described above is also an example of a mapping, which is not a connectivity or a peripherally continuous map, but is a transformation which carries connected subsets of $I$ onto connected subsets of $I$.

\section{REFERENCES}

1. John Nash, Generalized Brouwer theorem, Bull. Amer. Math. Soc. Research Problem 62-1-76.

2. Karol Borsuk, Quelques théorèmes sur les ensembles unicohêrents, Fund. Math. vol. 17 (1933) pp. 171-209.

3. Witold Hurewicz, and Henry Wallman, Dimension theory, Princeton, 1941.

4. R. L. Wilder, Topology of manifolds, New York, 1949.

Oklahoma State University 